Special issue of the International Conference on Computational and Experimental Science and Engineering (ICCESEN 2014)

\title{
LED Board Error Detection Automation with Image Processing
}

\author{
İ.S. ÜNCت̈̈ $\ddot{U}^{a, *}$ S. CoşKUNSU ${ }^{b}$ \\ ${ }^{a}$ Süleyman Demirel University Technology Faculty, Electrical and Electronic Engineering Department, \\ Isparta, Turkey \\ ${ }^{b}$ Süleyman Demirel University Engineering Faculty, Electronics and Communication Engineering Department, \\ Isparta, Turkey
}

\begin{abstract}
Nowadays, a great part of energy consumption belongs to lighting products. For this reason, lighting fixtures that consume less energy but perform more efficiently are manufactured. LEDs are the latest product of this development. LEDs have advantages over traditional lighting systems, such as being efficient, long lasting and environment friendly. Recently, with the development of technology, the image processing techniques are used in various fields. Image processing-based fault detection systems have become a frequently referenced solution in industrial automation systems due to the rapid analysis ability and high level of accuracy. Along with the use of cameras in the photometric measurements, it has become possible to make multiple measurements on the same photograph. In this study, control automation has been developed to determine the photometric properties in LED boards, used in the production of LED lighting fixtures. Improper LEDs are detected by checking colour and brightness of all LEDs on the LED board by means of a computer-controlled system.
\end{abstract}

DOI: 10.12693/APhysPolA.128.B-471

PACS: 85.60.Jb, 88.05.Tg

\section{Introduction}

Along with the rapid increase in the world population, energy efficiency has become increasingly important. The main objective in every new designed electronic device is to ensure the maximum efficiency with minimum energy consumption. $25 \%$ of the total electricity produced in Turkey is used in lighting [1]. Because of the fact that lighting has a great part of energy consumption, lighting luminaries that consume less energy but produce more efficient lighting have been manufactured.

With the development of semiconductor technology, lighting products having high efficiency lighting and broad colour portfolio have been obtained. LEDs are the product of this development [2]. Compared to conventional lighting systems, LEDs have several advantages, such as low energy consumption, longevity, strength, reduced-size, fast-switching, high durability and reliability [3]. Operation at low voltage, lack of warming problem and possibility to be located in a fully enclosed structure make the LEDs a perfect alternative solution for outdoor lighting, made with simple fixtures [4]. Besides, in the LED lighting systems it becomes possible to provide an energy saving of between $75 \%$ and $93 \%$ [1]. As a result of developments in the new generation of LED technology, as of the 2011 the LED lamps have reached the proficiency to be used instead of fluorescent lamps. In the forthcoming years along with the improvements

\footnotetext{
*corresponding author; e-mail: serkanuncu@sdu.edu.tr
}

in the parameters of luminous flux, continuity and efficiency, the use of a new generation of LED lamps will be inevitable in the entire lighting industry. This will entail the use of environmentally friendly light sources as well as power savings [3].

Because of their advantages over other lighting products, the usage of LEDs has increased; therefore, the number of companies producing LED is also expected to increase. For better quality production, the use of postproduction control mechanisms will be inevitable. Companies manufacturing LED lighting products will require this type of control mechanisms to detect error rates occurring in production and to identify the types of errors, to take measures accordingly.

LED lines are one of the products commonly used in LED lighting. LED lines are used in various fields from indoor lighting to outdoor wall lighting. LED lines are manufactured by grouping them into LED boards during the production phase (Fig. 1). After finishing the control of LED boards, the LED lines are formed by separating rows from each other.

Kumar et al. [5], measured the brightness by using high-resolution photographs. Brightness measurement was made with a calibrated camera. Calibration adjustment was set through luminance meter. Ismail et al. [6], have developed a webcam-based luminance meter. Images taken in a controlled environment were analyzed by image processing. Image processing results demonstrate a linear relationship between the mean grey value of the photos converted to gray-scale format and their illumination value. In his study, Hiscocks [7] used cameras to find out the total brightness of multiple LED photos. Exposure time is calculated with the camera calibration, 


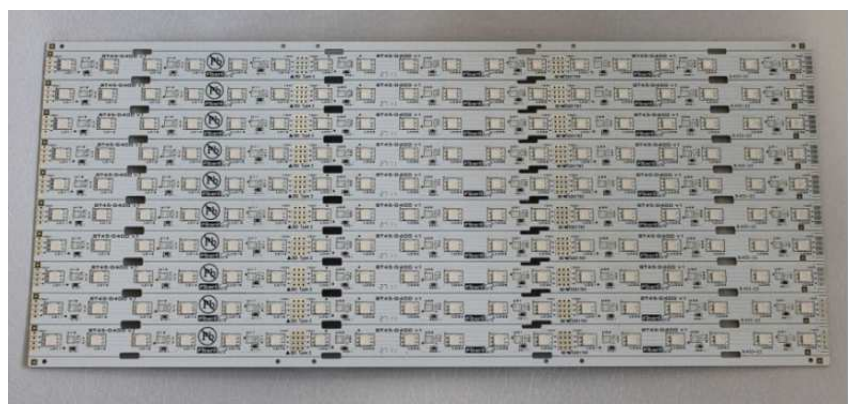

Fig. 1. LED board.

reference value of which is measured via luminance meter and total brightness of the photo is calculated by using camera properties such as the ISO setting.

In this study, using image processing techniques, the analysis of each LED on the LED board has been carried out. Analysis of both colour and brightness was performed. The main goal is to create a fast error control automation to minimize the margin of error. Another result obtained in this research is that with more simple synthetic image system, the image processing can be done in a quality manner.

\section{Methods and materials}

Measurement system has been developed for multiple LED photometric measurements. This system consists of two parts, namely software and hardware. In the software to determine the LED photometric properties, image processing algorithms have been developed. In the hardware part for accurate photometric measurements, a shooting pool, isolated from ambient light, is formed.

Flow-chart of the system operation is presented in Fig. 2. The diagram showing the hardware equipment is shown in Fig. 3.

An overview of the system is shown in Fig. 4. LED board being photometrically analyzed is located on the right side of the analysis table in the photo. Photographing pool required for analysis is integrated with the table. Pneumatic system ensuring fast and accurate analysis of the LED board is in the middle of the table. A compressor is providing compressed air to the pneumatic mechanism in the system. The computer connected to the system ensures the control of camera the hardware components and the electronic control card.

To perform the photometric analysis without being affected by ambient light, shooting pool is formed (Fig. 5). This pool includes camera, electronic control card and pneumatic system.

The software of the system has been developed with Visual Studio, in the C\# programming language. Software communicates with the control card and the camera and performs image processing, necessary for photometric measurements.

To perform the photometric analysis, photos were first converted to gray-scale format and then into "black and

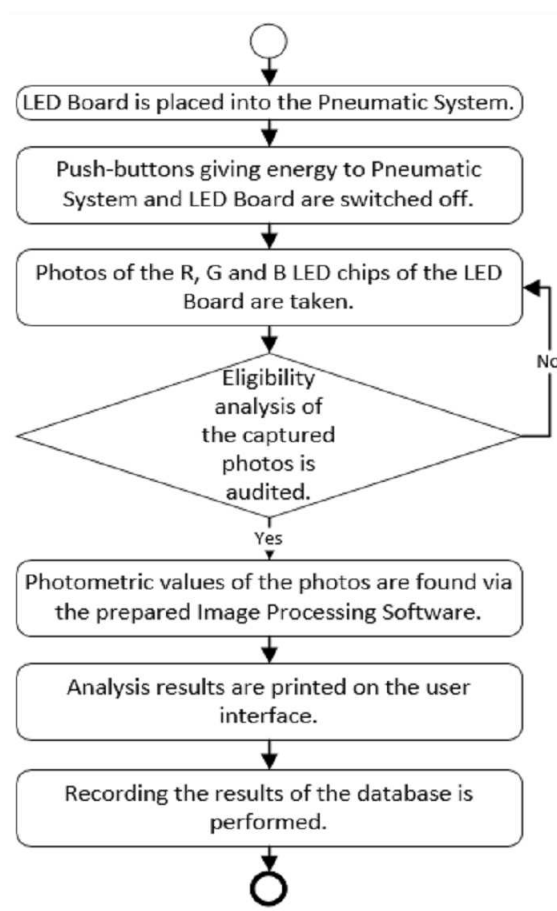

Fig. 2. Flow-chart of the system operation.

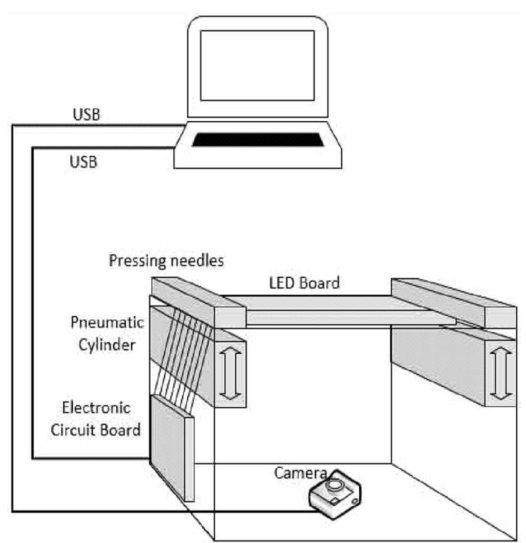

Fig. 3. Diagram showing the equipment.

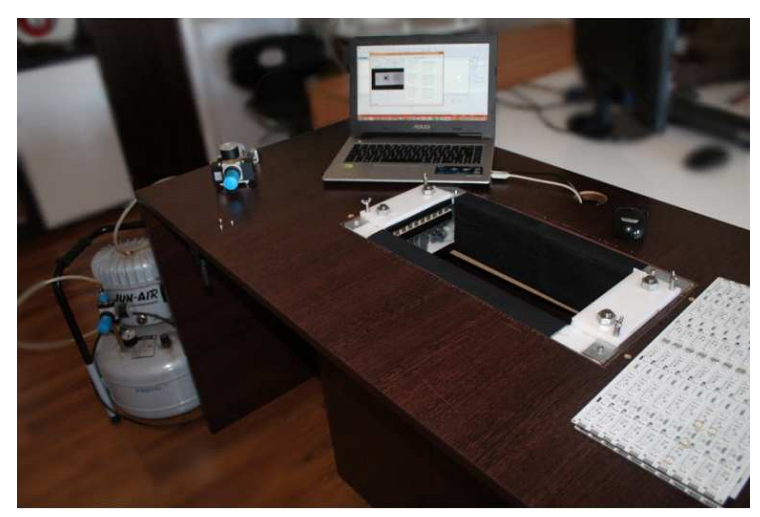

Fig. 4. Overview of the system. 


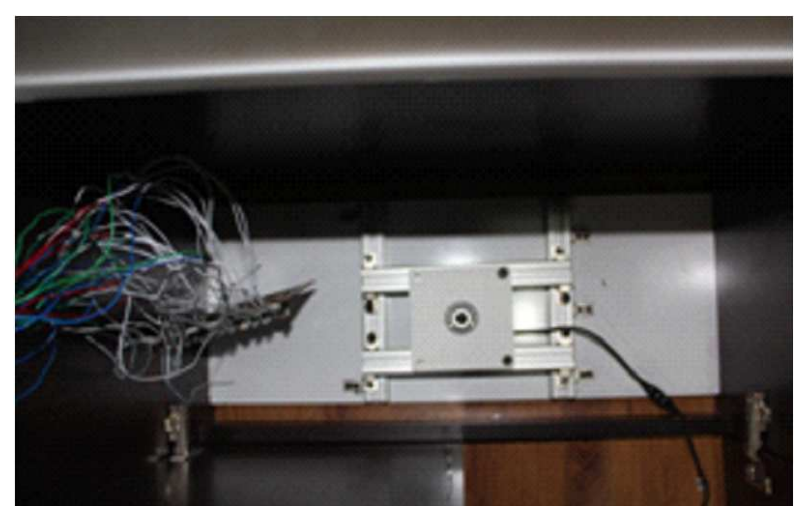

Fig. 5. Shooting pool.

white" format. Then, the location of each LED in the photo was identified by using LED detection algorithm based on morphological filters. The biggest advantage of LED finding algorithm is that it works independently of the number of LEDs in the LED board. Thus, the measurement of LED Board with a number of different LED can be made with the same algorithm without any adjustment.

In photometric analysis, brightness and colour controls for each LED in the LED Board is made separately. Image processing software analyses the results obtained from brightness analysis. The LED brightness value is between 0 and 255. According to the range of brightness, it is classified into four categories as high gloss, luminous, dim and non-luminous. Colour control is carried out in three primary colours (red, blue, green). The RGB colour space is used in the analysis. The faulty LEDs are marked and their presence is reported to the software users (Fig. 6).
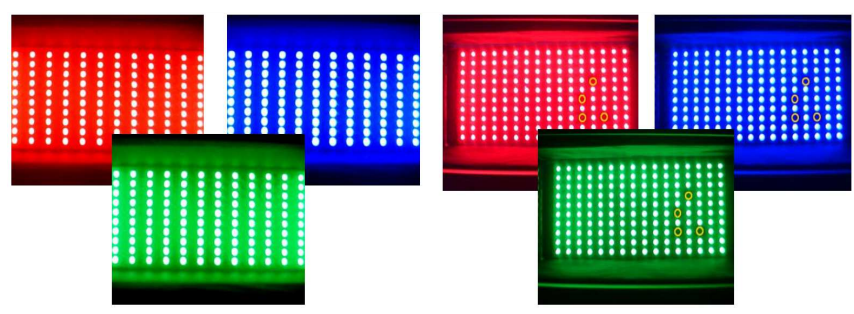

Fig. 6. The software outputs.

When the analysis is complete, the values are recorded automatically in the database. The recorded data are, a unique ID assigned to each LED board, analysis date and time, number of LEDs on the board, LED number for each class of brightness. This system can be used as a control mechanism at the end of the production line.

\section{Conclusions}

In our system, multiple LED measurements were carried out. Through analyzing the brightness and colour of each LED separately, the database record of the results obtained was performed. In this study it has been proven that the photometric measurements can be done economically. Photometric properties of the specified LED can be obtained numerically by using various image processing algorithms on photos taken from the camera in a controlled environment. These photometric information can be converted into such data as photometric variable light intensity, luminous level, colour temperature, colour rendering and brightness. The developed system can be also used as a control mechanism at the end of the mass production line.

Image processing, has introduced the concept of a real value assignment with photometric measurements. With this concept, by writing photometric values on the points in the area seen by the naked eyes, it becomes easy for the users to examine the changes in all areas of the photo on the screen simultaneously.

\section{Acknowledgments}

This work was supported in part by Scientific Research Projects Unit of Süleyman Demirel University (Project Number: 3351-YL1-12)

\section{References}

[1] C. Perdahçı, U. Hanlı, III. Energy Efficiency and Quality Symposium Proceedings, Kocaeli 2009, p. 323.

[2] MEGEP, Ministry of Education, Lighting Elements, 2008, retrieved 8 June 2012 from http://megep.meb.gov.tr/mte_program_modul/ modul_pdf/214T00028.pdf.

[3] Y. Erol, T. Canbolat, Electricity - Electronics and Computer Symposium, Elazığ 2011, p. 239.

[4] M.T. Gençoğlu, E. Özbay, XII. National Congress of Electricity, Electronics, Computer, Biomedical Engineering, Eskişehir 2007, p. 28.

[5] T.S. Kumar, C.P. Kurian, 2014 International Conference on Advances in Energy Conversion Technologies (ICAECT), IEEE, 2014, p. 193.

[6] A.H. Ismail, M.M. Azmi, M.A. Hashim, M.N. Ayob, M.S.M. Hashim, H.B. Hassrizal, IEEE Symposium on Computers 83 Informatics (ISCI), IEEE, 2013, p. 70.

[7] P.D. Hiscocks, Measuring Luminance with a digital camera, Syscomp Electronic Design Limited, www. syscompdesign. com, 2014. 